\title{
Assessing molecular-level compositional heterogeneity of natural organic matter in bentonites
}

\author{
MuHAMmed O. USMAN \& MYRnA J. SimPSON \\ University of Toronto, Toronto, Canada.
}

Sodium-rich bentonite clays have been chosen as the buffer material in the deep geological repository (DGR) concept of the Nuclear Waste Management Organisation (NWMO) plan for the long-term storage of used nuclear fuel. Characterisation of natural organic matter (NOM) in the bentonites reveals that NOM has the potential to serve as substrate for microbial activity. However, it is unclear if NOM is similar in composition within samples collected from different location from the same bentonite deposit and whether NOM chemistry vary within different products; thus, rendering the assessment of potential variability in the geochemistry of different bentonites from the same source crucial. Here we report the detailed geochemical characterisation of NOM in MX-80 bentonites from the same location (Wyoming) but sampled at different times at the bulk and molecular level. Our results show that total carbon is less than $1 \%(0.70 \pm 0.04 \%)$ and organic carbon constitutes less than $20 \%(0.10 \pm 0.01 \%)$ of the total carbon content. Statistical analyses revealed no significant difference in the bulk NOM composition. Targeted organic compound analyses indicate a predominance of long-chain homologues among the analysed compound classes. We detected nanogramlevel variabilities in selected compounds among the samples. However, these variabilities are not statistically significant (at $\mathrm{P} \leq 0.05$ ). Solid-state ${ }^{13} \mathrm{C}$ NMR analysis showed consistency between the different samples and the predominance of aliphatic as well as aromatic compounds which suggests that the NOM has undergone extensive degradation. Overall, all analyses are consistent and indicate that the NOM composition exhibits low heterogeneity in different bentonite samples. 